\author{
ANNALES \\ POLONICI MATHEMATICI \\ XXXII (1976)
}

\title{
Higher order singular perturbation method for linear differential equations in Banach spaces
}

\author{
by JANUsz Mrka (Swierk, Otwock)
}

\begin{abstract}
The singular perturbation method is developed rigorously for evolution equations in Banach spaces with a small positive parameter multiplying the time derivative. The asymptotic solution uniformly convergent to the exact solution up to an arbitrary order in the small parameter consists of the inner and outer asymptotio solutions. The present approach is compared with and shown to be simpler than the standard one, based upon the formal expansions of the functions and operators involved into the power or Taylor series.
\end{abstract}

Introduction. The singular perturbation method is an important tool in the practical analysis of various equations of mathematical physics. In many cases the method has been applied without a rigourous mathematical justification and the formulas derived from intuitive arguments. A comprehensive account of such an approach is given by Cole [1].

Side by side with practical applications the singular perturbation method has also been analyzed in a rigorous manner for various types of differential equations. A survey of the results obtained in this field is given by O'Malley [9].

An important domain of application of the singular perturbation method are the evolution equations in a Banach space with the time derivative multiplied by a small positive parameter. Such equations were first considered by Krein [8], who showed that, if proper conditions are satisfied, the asymptotic solution of the zero order tends to the exact solution uniformly in time.

In the previous papers [10], [11] the author has proved the same result for a system of evolution equations equivalent to a single evolution equation containing additional integral terms. He has also shown that such an approach can be applied to the linear transport equation for which the singular perturbation method has been introduced formally by Bell and Hendry [5] and by Hendry [3], [4].

In this paper the higher order singular perturbation method will be rigorously analyzed for evolution equations in a Banach space. It will be shown that under proper conditions the asymptotic solution of any 
finite order tends, in a suitably defined sense, uniformly with respect. to time, to the exact solution.

The perturbation procedure applied in this paper leads to slightly simpler equations than those obtained by means of the standard procedure. The two approaches are compared in the last section of the paper.

The results known from literature are quoted without proofs. For details see References [2], [6]-[8].

Equations of evolution in a Banach space. Let $E$ be a complex Banach space with the norm $\|\cdot\|$.

Definition 1. The family $\{G(t) ; 0 \leqslant t\}$ of bounded operators in $E$ will be called a strongly continuous semigroup if

(i) $G(t+s)=G(t) G(s) ; 0 \leqslant t ; 0 \leqslant 8$;

(ii) $\boldsymbol{G ( 0 )}=I$;

(iii) for each $x \in E$ the function $t \rightarrow G(t) x$ is strongly continuous on $[0, \infty)$.

Definition 2. The operator

$$
A x=\lim _{t \rightarrow 0} \frac{1}{t}[G(t) x-x],
$$

with the domain $D(A)$ consisting of all $x \in E$ such that the limit exists in the norm in $E$, is called a generator of a strongly continuous semigroup. $\{G(t) ; 0 \leqslant t\}$.

LEMMA 1. The operator $A$ from Definition 2 is a closed linear operator. and its domain $D(A)$ is dense in $E$.

If $x \in D(A)$, then for each $t \in[0, \infty)$ the element $G(t) x$ belongs to $D(A)$ and the function $t \rightarrow G(t) x$ is strongly continuously differentiable on $[0, \infty)$. such that

$$
\left.\frac{d}{d t} f(t) x\right)=A G(t) x=G(t) A x .
$$

THEOREM 1 (Hillo-Yosida). A necessary. and sufficient condition that a closed linear operator $A$ with the domain $D(A)$ dense in $E$ be a generator of a strongly continuous semigroup $\{G(t) ; 0 \leqslant t\}$ of bounded operators such. that for each $t \in[0, \infty)$

$$
\|G(t)\| \leqslant \exp (\omega t)
$$

for some real $\omega$ is that the resolvent of $A$

$$
R(\lambda, A)=(\lambda I-A)^{-1}
$$

should exist for each $\lambda>\omega$ and should be an operator defined on the whole: space $E$, with the norm satisfying the inequality

$$
\|R(\lambda, A)\| \leqslant(\lambda-\omega)^{-1} \text {. }
$$


LEMMA 2. If the operator $A$ satisfies the requirements of Theorem 1 and the function $t \rightarrow q(t)$ with the values from $E$ is strongly continuously differentiable on $[0, T]$, where $T$ is a fixed positive number, then the evolution equation

$$
\frac{d}{d t}(x(t))=A x(t)+q(t)
$$

with the initial condition

$$
x(\mathbf{0})=\eta \in D(A)
$$

has on $[0, T]$ a unique strongly continuously differentiable solution $\{x(t)$; $0 \leqslant t \leqslant T\}$ such that

$$
x(t)=\theta(t) \eta+\int_{0}^{t} d s G(t-s) q(s) .
$$

The integral is understood as a strong limit of Riemann sums. The same meaning will be attached to other integrals of abstract functions appearing in this paper.

LEMMA 3. If the operator $A$ satisfies the requirements of Theorem 1 and 80 is a generator of a strongly continuous semigroup $\{G(t) ; 0 \leqslant t\}$, then the operator $\frac{1}{\varepsilon} A$, where $\varepsilon>0$, is a generator of the semigroup $\{G(t / \varepsilon) ; 0 \leqslant t\}$. If the function $t \rightarrow q(t)$ is strongly continuously differentiable on $[0, T]$, then the evolution equation

$$
\varepsilon \frac{d}{d t}\left(x_{s}(t)\right)=A x_{e}(t)+q(t)
$$

with the initial condition

$$
x_{\bullet}(0)=\eta \in D(A)
$$

has on $[0, T]$ a unique strongly differentiable solution $\left\{x_{\theta}(t) ; 0 \leqslant t \leqslant T\right\}$ such that

$$
x_{s}(t)=G\left(\frac{t}{\varepsilon}\right) \eta+\frac{1}{\varepsilon} \int_{0}^{t} d s G\left(\frac{t-s}{\varepsilon}\right) q(s) .
$$

Definition 3. A family $\{U(t, s) ; 0 \leqslant s \leqslant t \leqslant T\}$ of bounded operators in $E$ will be called a strongly continuous quasi-semigroup if

(i) $U(t, s)=U(t, \tau) U(\tau, s) ; 0 \leqslant 8 \leqslant \tau \leqslant t \leqslant T$;

(ii) $U(t, t)=I ; 0 \leqslant t \leqslant T$;

(iii) for each $x \in E$ the function $t, s \rightarrow U(t, s) x$ is strongly continuous on the triangle $0 \leqslant 8 \leqslant t \leqslant T$. 
THEOREM 2. Let $\{A(t) ; 0 \leqslant t \leqslant T\}$ be a family of closed operators in $E$ such that

(i) for each $t \in[0, T]$ the domain $D(A)$ of the operator $A(t)$ is independent of $t$ and $D(A)$ is dense in $E$;

(ii) for eaoh $\tau \in[0, T]$ the operator $A(\tau)$ is a generator of a strongly continuous semigroup $\left\{G_{\tau}(t) ; 0 \leqslant t\right\}$ such that for each $t \in[0, \infty)$

and

$$
\left\|G_{v}(t)\right\| \leqslant \exp \left(a_{\tau} t\right)
$$

$$
a=\sup _{\tau \in[0, T]} a_{\tau}<0 \text {; }
$$

(iii) for each $x \in D(A)$ the function $t \rightarrow A(t) x$ is strongly continuously differentiable on $[0, T]$.

Then the family $\{A(t) ; 0 \leqslant t \leqslant T\}$ is a generator of a strongly continuous quasi-semigroup $\{U(t, s) ; 0 \leqslant 8 \leqslant t \leqslant T\}$ in the sense that for $x \in D(A)$ and $0 \leqslant s \leqslant t \leqslant T$ we have $U(t, s) x \in D(A)$, the function $t, 8 \rightarrow U(t, s) x$ is strongly continuously differentiable on the triangle $0 \leqslant s \leqslant t \leqslant T$, and the following identities are valid:

$$
\frac{\partial}{\partial t}(U(t, s) x)=A(t) U(t, s) x ; \quad \frac{\partial}{\partial s}(U(t, s) x)=-U(t, s) A(s) x .
$$
integral

The quasi-semigroup may be expressed for any $x \in E$ by the multiplicative

$$
U(t, 8) x=\lim G_{\tau_{n}}\left(t_{n}-t_{n-1}\right) G_{\tau_{n-1}}\left(t_{n-1}-t_{n-2}\right) \ldots G_{\tau_{1}}\left(t_{1}-t_{0}\right) x,
$$

where $8=t_{0}<t_{1}<\ldots<t_{n}=t$ are the points of divisions of the integral $[8, t]$ and $t_{i-1}<\tau_{i}<t_{i}$. The limit is understood in the strong sense for $\max \left(t_{i}-t_{i-1}\right) \rightarrow 0$. For $0 \leqslant 8 \leqslant t \leqslant T$ the norm of $U(t, 8)$ satisfies the inequality $\|U(t, s)\| \leqslant \exp (a(t-8))$.

LFMMA 4. If the family $\{A(t) ; 0 \leqslant t \leqslant T\}$ satisfies the requirements of Theorem 2 and is a generator of a strongly continuous quasi-semigroup $\{U(t, s) ; 0 \leqslant 8 \leqslant t \leqslant T\}$ and the function $t \rightarrow q(t)$ is strongly continuously differentiable on $[0, T]$, then the evolution equation

$$
\frac{d}{d t}(x(t))=A(t) x(t)+q(t)
$$

with the initial condition

$$
x(0)=\eta \in D(A)
$$

has on $[0, T]$ a unique strongly differentiable solution $\{x(t) ; 0 \leqslant t \leqslant T\}$ such that

$$
x(t)=U(t, 0) \eta+\int_{0}^{t} d s U(t, s) q(s)
$$


LEMma 5. If the family $\{A(t) ; 0 \leqslant t \leqslant T\}$ satisfies the requirements of Theorem 2 and is a generator of a strongly continuous quasi-semigroup $\{U(t, 8) ; 0 \leqslant 8 \leqslant t \leqslant T\}$, then the family $\left\{\frac{1}{\varepsilon} A(t) ; 0 \leqslant t \leqslant T\right\}$, where $\varepsilon>0$, is a generator of a strongly continuous quasi-semigroup $\left\{U_{8}(t, s) ; 0 \leqslant 8\right.$ $\leqslant t \leqslant T\}$. For $0 \leqslant 8 \leqslant t \leqslant T$ the norm of $U_{0}(t, s)$ satisfies the inequality

$$
\left\|U_{\varepsilon}(t, s)\right\| \leqslant \exp \left(\frac{a}{\varepsilon}(t-s)\right)
$$

where a is defined in Theorem 2.

If the function $t \rightarrow q(t)$ is strongly continuously differentiable on $[0, T]$, then the evolution equation

$$
\varepsilon \frac{d}{d t}\left(x_{e}(t)\right)=A(t) x_{8}(t)+q(t)
$$

with the initial condition

$$
x_{s}(0)=\eta \in D(A)
$$

has on $[0, T]$ a unique strongly differentiable solution $\left\{X_{8}(t) ; 0 \leqslant t \leqslant T\right\}$ such that

$$
x_{\varepsilon}(t)=U_{s}(t, 0) \eta+\frac{1}{\varepsilon} \int_{0}^{t} d s U_{\bullet}(t, s) q(s) .
$$

Asymptotic solutions to the evolution equation. In this section it will be assumed that the family $\{A(t) ; 0 \leqslant t \leqslant T\}$ and the function $t \rightarrow q(t)$ considered previously have some additional properties.

Assumption 1. The family $\{A(t) ; 0 \leqslant t \leqslant T\}$ satisfies the requirements of Theorem 2. Additionally, for each $t \in[0, T]$ and $x \in D(A)$ the operator $A(t)$ can be expressed in the form

$$
A(t) x=A_{0} x+A_{1}(t) x,
$$

where $A_{0}$ is a closed operator independent of $t$ with the domain $D(A)$ dense in $E$ and $\left\{A_{1}(t) ; 0 \leqslant t \leqslant T\right\}$ is a family of bounded operators defined for all $x \in E$ and such that the funotion $t \rightarrow A_{1}(t)$ is $(N+1)$ times uniformly continuously differentiable on $[0, T]$. The funotion $t \rightarrow q(t)$ is $(N+1)$ times strongly continuously differentiable on $[0, T]$.

LEMMA 6. If the family $\{A(t) ; 0 \leqslant t \leqslant T\}$ and the function $t \rightarrow q(t)$ satisfy Assumption 1, then the solution $\left\{x_{c}(t) ; 0 \leqslant t \leqslant T\right\}$ of the evolution oquation

$$
\left.\varepsilon \frac{d}{d t} \mid x_{\bullet}(t)\right)=A(t) x_{s}(t)+q(t)
$$


with the initial condition

$$
x_{\varepsilon}(0)=\eta \in D(A) \text {. }
$$

is such that $x_{s}(t)$ can be written as

$$
x_{s}(t)=\bar{x}_{a}^{(N)}(t)+U_{s}(t, 0)\left(\eta-\bar{x}_{s}^{(N)}(0)\right)-\varepsilon^{N} \int_{0}^{t} d s U_{0}(t, s) \frac{d}{d s}\left(\bar{x}_{N}(s)\right),
$$

where

$$
\begin{gathered}
\bar{x}_{\varepsilon}^{(N)}(t)=\sum_{n=0}^{N} \varepsilon^{n} \bar{x}_{n}(t) ; \\
\bar{x}_{0}(t)=-A^{-1}(t) q(t) ; \quad \bar{x}_{n}(t)=A^{-1}(t) \frac{d}{d t}\left(\bar{x}_{n-1}(t)\right) ; \quad n=1,2, \ldots, N .
\end{gathered}
$$

The family $\left\{\bar{x}_{s}^{(N)}(t) ; 0 \leqslant t \leqslant T\right\}$ is called the outer asymptotic solution of order $N$.

Proof. The integral term in the expression for $x_{8}(t)$ can be integrated by parts:

$$
\begin{aligned}
& \frac{1}{\varepsilon} \int_{0}^{t} d s U_{\varepsilon}(t, s) q(8)=\frac{1}{\varepsilon} \int_{0}^{t} d s U_{s}(t, s) A(s) A^{-1}(s) q(8) \\
& =-\frac{1}{\varepsilon} \int_{0}^{t} d s U_{8}(t, s) A(s) \bar{x}_{0}(s)=\int_{0}^{t} d s \frac{\partial}{\partial s}\left(U_{0}(t, s)\right) \bar{x}_{0}(8) \\
& =\bar{x}_{0}(t)-U_{s}(t, 0) \bar{x}_{0}(0)-\int_{0}^{t} d s U_{s}(t, s) \frac{d}{d s}\left(\bar{x}_{0}(s)\right) \\
& =\bar{x}_{0}(t)-U_{\theta}(t, 0) \bar{x}_{0}(0)-\varepsilon \cdot \frac{1}{\dot{\varepsilon}} \int_{0}^{t} d s U_{s}(t, 8) A(8) \bar{x}_{1}(8) \text {. }
\end{aligned}
$$

The validity of the above manipulations stems from the assumed properties of the family $\{A(t) ; 0 \leqslant t \leqslant T\}$ and the function $t \rightarrow q(t)$ and from the formula

$$
\frac{\partial}{\partial s}\left(U_{\varepsilon}(t, s)\right) x=-\frac{1}{\varepsilon} U_{\varepsilon}(t, 8) A(s) x ; x \in D(A)
$$

implied by Theorem 2.

Repeating $N$ times the differentiation by parts, one gets the final expression for $x_{e}(t)$. QED.

LEMMA 7. The solution $\left\{x_{s}(t) ; 0 \leqslant t \leqslant T\right\}$ of the evolution equation of Lemma 1 is such that, as $\varepsilon$ tends to zero, the function $t \rightarrow x_{s}(t)$ tends uniformly to the function

$$
t \rightarrow \bar{x}_{a}^{(N)}(t)+U_{\bullet}(t, 0)\left(\eta-\bar{x}_{a}^{(N)}(0)\right)
$$


faster than $\varepsilon^{N}$. In other words, the function

$$
t \rightarrow \varepsilon^{-N}\left(x_{s}(t)-\bar{x}_{s}^{(N)}(t)\right)-U_{s}(t, 0)\left(\eta-\bar{x}^{(N)}(0)\right)
$$

tends to zero uniformly on $[0, T]$.

Proof. The integral term in the expression for $x_{e}(t)$ satisfies the inequality

$$
\begin{aligned}
& \left\|\varepsilon_{N} \int_{0}^{t} d s U_{s}(t, s) \frac{d}{d s}\left(\bar{x}_{N}(s)\right)\right\| \\
& \quad \leqslant \varepsilon^{N+1} \cdot M \cdot \frac{1}{\varepsilon} \int_{0}^{t} d s \exp \left(\frac{a}{\varepsilon}(t-8)\right)=\varepsilon^{N+1} \cdot M\left(1-\exp \left(\frac{\alpha}{\varepsilon} t\right)\right),
\end{aligned}
$$

where

$$
M=\sup _{t \in[0, T]}\left\|\frac{d}{d t}\left(\bar{x}_{N}(t)\right)\right\|
$$

and $M<\infty$ since the function $t \rightarrow \frac{d}{d t}\left(\bar{x}_{N}(t)\right)$ is uniformly bounded on $[0, T]$. According to the requirements of Theorem $2 a<0$, so that the integral term tends to zero in the norm with $\varepsilon \rightarrow 0$ uniformly on $[0, T]$. QED.

COROLLARY 1. Replacing the family $\{A(t) ; 0 \leqslant t \leqslant T\}$ and the quasisemigroup $\{U(t, s) ; 0 \leqslant 8 \leqslant t \leqslant T\}$ in Lemmas 6 and 7 by the operator $A$ satisfying the requirements of Theorem 1 and by the semigroup $\{G(t) ; 0 \leqslant t\}$, respectively, one can obtain the corresponding results for the operators explicitly independent of time.

Definition 4. An abstract function $\left\{\varphi_{0}(t) ; 0 \leqslant t \leqslant T ; 0<\varepsilon\right\}$ will be said to tend to zero $\beta$-nearly uniformly on $(0, T]$ if for each $\delta>0$ and each $\beta \epsilon(0,1)$ there exists $a, K>0$ and $\varepsilon_{0}>0$ such that for $0<\varepsilon \leqslant \varepsilon_{0}$ and $K \varepsilon^{\beta} \leqslant t \leqslant T$

$$
\left\|\varphi_{.}(t)\right\|<\delta .
$$

If the above statements are valid for each $\beta \epsilon(0,1]$, then the function $\left\{\varphi_{s}(t) ; 0 \leqslant t \leqslant T ; 0<\varepsilon\right\}$ is said to tend to zero nearly uniformly on $(0, T]$.

It is seen that if $\varphi_{\varepsilon}(t)=\exp (-a t / \varepsilon) \cdot g$ for any $a>0$ and $g \epsilon E$, then the family $\left\{\varphi_{\varepsilon}(t) ; 0 \leqslant t \leqslant T ; 0<\varepsilon\right\}$ tends to zero nearly uniformly on $(0, T]$ and if $\varphi_{\varepsilon}(t)=\varepsilon^{\gamma} \cdot \exp (-a t / \varepsilon) \cdot g$ for any $\gamma<0, a>0$, and $g \in E$, then the family $\left\{\varphi_{s}(t) ; 0 \leqslant t \leqslant T ; 0<\varepsilon\right\}$ tends to zero $\beta$-nearly uniformly on $(0, T]$. Definition 4 is an adaptation of the definition introduced by Krein ([8]; Definition 1.1, Chapter IV).

LEMMA 8. If the operator $A$ satisfies the requirements of Theorem 1 with $\omega<0$ and is a generator of a strongly continuous semigroup $\{G(t) ; 0 \leqslant t\}$, 
then the family $\{G(t / \varepsilon) ; 0 \leqslant t \leqslant T\}$ tends to zero nearly uniformly on $(0, T]$ and the family $\left\{\varepsilon^{\gamma} G(t / \varepsilon) ; 0 \leqslant t \leqslant T\right\}$ for $\gamma<0$ tends to zero $\beta$-nearly uniformly on $(0, T]$.

Similarly, if the family $\{A(t) ; 0 \leqslant t \leqslant T\}$ satigfies the requirements of Theorem 2 and is a generator of a strongly continuous quasi-semigroup $\{U(t, 8) ; 0 \leqslant s \leqslant t \leqslant T\}$, then the family $\left\{U_{8}(t, s) ; 0 \leqslant 8 \leqslant t \leqslant T\right\}$ tends to zero nearly uniformly on $(8, T]^{\bullet}$ and the family $\left\{\varepsilon^{\gamma} U_{.}(t, 8) ; 0 \leqslant s \leqslant t \leqslant T\right\}$ for $\gamma<0$ tends to zero. $\beta$-nearly uniformly on $(\mathcal{S}, T]$.

Proof. The lemma follows directly from Lemma 3 and Lemma 5. QED.

COROLLARY 2. It follows from Lemmas 7 and 8 that the outer asymptotic solution $\left\{\bar{x}_{a}^{(N)}(t) ; 0 \leqslant t \leqslant T\right\}$ tends to the exact solution $\left\{x_{e}(t) ; 0 \leqslant t \leqslant T\right\}$ nearly uniformly on $(0, T]$.

Definition 5. Let the quasi-semigroup $\left\{U_{\imath}(t, 8) ; 0 \leqslant 8 \leqslant t \leqslant T\right\}$ tends to zero nearly uniformly on $(0, T]$. Then the family of bounded operators $\left\{U_{0}^{(N)}(t, 8) ; 0 \leqslant 8 \leqslant t \leqslant T\right\}$ is said to approximate $\left\{U_{0}(t, 8)\right.$; $0 \leqslant s \leqslant t \leqslant T\}$ up to the order $N$ if the function $t \rightarrow \varepsilon^{-N}\left(U_{c}(t, 8) g-U_{0}^{(N)}(t, s) g\right)$ tends to zero uniformly with respect to $t$ on the interval $[8, T]$ for any $8 \in[0, T]$ and $g \in D(A)$. In other words, for any $g \in D(A), 8 \in[0, T]$ and $\delta>0$ there exists an $\varepsilon_{1}>0$ such that for $0<\varepsilon \leqslant \varepsilon_{1}$ and $8 \leqslant t \leqslant T$

$$
\varepsilon^{-N}\left\|U_{.}(t, s) g-D_{e}^{(N)}(t, s) g\right\|<\delta .
$$

Definition 6. The family $\left\{U_{e}^{(N)}(t, 0)\left(\eta-\bar{x}^{(N)}(0)\right) ; 0 \leqslant t \leqslant T\right\}$ will be called the inner asymptotic solution of the order $N$ of the evolution equation

$$
\varepsilon \frac{d}{d t}\left(x_{\varepsilon}(t)\right)=A(t) x_{\theta}(t)+q(t)
$$

with the initial condition

$$
x_{8}(0)=\eta \in D(A) \text {. }
$$

LEMMA 9. The family $\left\{U_{8}^{(N)}(t, 8) ; 0 \leqslant s \leqslant t \leqslant T\right\}$ approximating up to the order $N$ the quasi-semigroup corresponding to the family $\{A(t) ; 0 \leqslant t$ $\leqslant T$ \} satisfying Assumption 1 can be defined for any $g \in D(A)$, as

$$
U_{s}^{(N)}(t, s) g=\sum_{n=0}^{N} \varepsilon^{n} y_{s}^{(n)}(t, 8),
$$

where $\left\{y_{\circ}^{(0)}(t, 8), y_{s}^{(1)}(t, 8), \ldots, y_{s}^{(N)}(t, 8) ; 0 \leqslant 8 \leqslant t \leqslant N\right\}$ is the solution of the system of evolution equations

$$
\begin{array}{r}
\varepsilon \frac{d}{d t}\left(y_{\varepsilon}^{(n)}(t, s)\right)=A(8) y_{a}^{(n)}(t, s)+\sum_{k=1}^{n} \frac{1}{k !}\left(\frac{t-8}{\varepsilon}\right)^{k} \frac{d^{k}}{d s^{k}}(A(s)) y_{\bullet}^{(n-k)}(t, 8) \\
n=0,1, \ldots, N
\end{array}
$$


with the initial condition

$$
y_{c}^{(0)}(s, 8)=g ; \quad y_{a}^{(1)}(s, s)=\ldots=y_{0}^{(N)}(s, s)=0 ; \quad 0 \leqslant s \leqslant T .
$$

Proof. It follows from Lemma 3 that for a fixed $s \in[0, T)$ the solution $\left\{y_{6}^{(0)}(t, 8) ; 0 \leqslant 8 \leqslant t \leqslant T\right\}$ of the first equation for $n=0$ is such that

$$
y_{\varepsilon}^{(0)}(t, 8)=G_{s}\left(\frac{t-8}{\varepsilon}\right) g,
$$

where the semigroup $\left\{G_{\theta}(\tau) ; 0 \leqslant \tau<\infty\right\}$ is generated by the operator $A(s)$. The function $t \rightarrow y_{\varepsilon}^{(0)}(t, s)$ is uniformly bounded on $[s, T]$. Since the operators $\theta_{s}\left(\frac{t-s}{\varepsilon}\right)$ obey on the triangle $0 \leqslant s \leqslant t \leqslant T$ the inequality

$$
\left\|G_{s}\left(\frac{t-8}{\varepsilon}\right)\right\| \leqslant \exp \left(\frac{a}{\varepsilon}(t-8)\right)
$$

with $a<0$, the function $t \rightarrow e^{-N} y_{:}^{(0)}(t, 8)$ tends to zero $\beta$-nearly uniformly on $(s, T]$.

The solution $\left\{y_{\varepsilon}^{(1)}(t, 8) ; 0 \leqslant s \leqslant t \leqslant T\right\}$ of the second equation for $n=1$ is such that

$$
y_{s}^{(1)}(t, s)=\frac{1}{\varepsilon} \int_{s}^{t} d s^{\prime} G_{s}\left(\frac{t-s^{\prime}}{\varepsilon}\right)\left(8^{\prime}-8\right) \frac{d}{d s}(A(s)) G_{s}\left(\frac{s^{\prime}-8}{\varepsilon}\right) g .
$$

The function $t \rightarrow y_{e}^{(1)}(t, 8)$ is uniformly bounded on $[8, T]$ and on the triangle $0 \leqslant 8 \leqslant t \leqslant T$

$$
\left\|y_{e}^{(1)}(t, s)\right\| \leqslant \exp \left(\frac{a}{\varepsilon}(t-8)\right) \frac{(t-8)^{2}}{2 \varepsilon} \cdot\left\|\frac{d}{d s}(A(s))\right\| \cdot\|g\| .
$$

This shows that the function $t \rightarrow \varepsilon^{-N+1} y_{a}^{(1)}(t, 8)$ tends to zero $\beta$-nearly uniformly on $(8, T]$.

Repeating the above reasoning $N$ times, one concludes finally that the family $\left\{\varepsilon^{-N} U_{s}^{(N)}(t, s) g ; 0 \leqslant s \leqslant t \leqslant T\right\}$, where

$$
D_{e}^{(N)}(t, s) g=\sum_{n=0}^{N} \varepsilon^{n} y_{s}^{(n)}(t, s)
$$

for $g \in D(A)$, is uniformly bounded on $[s, T]$ and tends to zero $\beta$-nearly uniformly on $(s, T]$.

Let $\left\{y_{s}(t, s) ; 0 \leqslant 8 \leqslant t \leqslant T\right\}$ be the solution of the evolution equation

$$
\varepsilon \frac{d}{d t}\left(y_{\cdot}(t, s)\right)=A(t) y_{\circ}(t, s)
$$

with the initial condition

$$
y_{s}(s, s)=g \in D(A) ; \quad 0 \leqslant s<T .
$$


Then the family $\left\{v_{s}^{(N)}(t, 8) ; 0 \leqslant 8 \leqslant t \leqslant T\right\}$ such that

$$
\begin{aligned}
v_{a}^{(N)}(t, 8) & =\varepsilon^{-N}\left(y_{a}(t, 8)-\sum_{n=0}^{N} \varepsilon^{n} y_{c}^{(n)}(t, 8)\right) \\
& =\varepsilon^{-N}\left(U_{\bullet}(t, 8) g-D_{c}^{(N)}(t, 8) g\right)
\end{aligned}
$$

is the solution of the evolution equation

$$
\varepsilon \frac{d}{d t}\left(v_{a}^{(N)}(t, s)\right)=A(t) v_{a}^{(N)}(t, s)+P_{c}^{(N)}(t, s)
$$

with the initial condition

$$
v_{8}^{(N)}(8,8)=0 ;
$$

The non-homogeneous term has the from

$$
P_{s}^{(N)}(t, s)=\sum_{n=0}^{N} \varepsilon^{-N+n}\left(A(t)-\sum_{m=0}^{N-n} \frac{(t-8)^{m}}{m !} \frac{d^{m}}{d s^{m}}(A(s)) y_{e}^{(n)}(t, s)\right),
$$

so that $v_{s}^{(N)}(t, 8)$ can be written as the sum

$$
v_{o}^{(N)}(t, 8)=\sum_{n=0}^{N} v_{\varepsilon}^{(N, n)}(t, 8),
$$

where

$$
\begin{aligned}
& v_{s}^{(N, n)}(t, s) \\
& =\varepsilon^{-N+n-1} \int_{a}^{t} d s^{\prime} U_{s}\left(t, s^{\prime}\right)\left(A\left(s^{\prime}\right)-\sum_{m=0}^{N-n} \frac{\left(8^{\prime}-8\right)^{m}}{m !} \frac{d^{m}}{d s^{m}}(A(s))\right) y_{s}^{(n)}\left(s^{\prime}, s\right) .
\end{aligned}
$$

It is seen from Assumption 1 that for all $g \in D(A)$

$$
\begin{aligned}
A^{(N-n)}(t, s) g & \equiv A(t) g-\sum_{m=0}^{N-n} \frac{(t-s)^{m}}{m !} \frac{d^{m}}{d s^{m}}(A(s)) g \\
& =A_{1}(t) g-\sum_{m=0}^{N-n} \frac{(t-8)^{m}}{m !} \frac{d^{m}}{d s^{m}}\left(A_{1}(s)\right) g
\end{aligned}
$$

This shows that $\left\{A^{(N-n)}(t, 8) ; 0 \leqslant 8 \leqslant t \leqslant T\right\}$ is a uniformly bounded family of operators.

The quasi-semigroup $\left\{U_{.}(t, 8) ; 0 \leqslant 8 \leqslant t \leqslant T\right\}$ satisfies the inequality

$$
\left\|U_{\bullet}(t, 8)\right\| \leqslant \exp \left(\frac{a}{\varepsilon}(t-8)\right) ; \quad 0 \leqslant 8 \leqslant t \leqslant T,
$$


which gives for

$$
f_{\varepsilon}^{(N, n)}(t, s)=\left\|v_{\varepsilon}^{(N, n)}(t, s)\right\|
$$

the following inequality:

$$
f_{o}^{(N, n)}(t, s) \leqslant \frac{1}{|a|} \varepsilon^{-N+n} \sup _{s^{\prime} \in[s, l]}\left(\left\|A^{(N-n)}\left(s^{\prime}, s\right)\right\| \cdot\left\|y_{s}^{(n)}\left(s^{\prime}, s\right)\right\|\right) .
$$

Consider $t \rightarrow A^{(N-n)}(t)$ as the function in the Banach space $L(E, E)$ of bounded operators defined on $E$. For such a function the Taylor expression can be introduced. In particular, one has

$$
\sup _{s^{\prime} \in[s, t]}\left\|A^{(N-n)}\left(s^{\prime}, s\right)\right\| \leqslant \frac{t^{N-n+1}}{(N-n+1) !} \sup _{s^{\prime} \in[s, l]}\left\|\frac{d^{N-n+1}}{d s^{\prime N-n+1}}\left(A_{1}\left(s^{\prime}\right)\right)\right\| \text {. }
$$

Finally, using the fact that the function $t \rightarrow y^{(n)}(t, s)$ is uniformly bounded on $[s, T]$, one gets the inequality

$$
f_{\varepsilon}^{(N, n)}(t, 8) \leqslant M \varepsilon^{N-n} t^{N-n+1}
$$

with a constant $M$ independent of $t$ and $\varepsilon$.

Let $t_{2}$ be a fixed point such that $8<t_{2} \leqslant T$ and $0<\beta \leqslant 1$. Then the function $f_{e}^{(N, n)}(t, 8)$ attains its maximum value in the interval $\left[8, \varepsilon^{\beta} t_{2}\right]$ at $t=\varepsilon^{\beta} t_{2}$. Thus

$$
f_{s}^{(N, n)}(t, s) \leqslant M \cdot t_{2}^{N-n+1} \cdot \varepsilon^{\beta(N-n+1)-N+n} .
$$

This shows that if $\beta>(N-n) /(N-n+1)$, then for each $\delta>0$ and $t_{2} \epsilon(s, T]$ there exists an $\varepsilon_{2}>0$ such that for $0<\varepsilon \leqslant \varepsilon_{2}$ and $s \leqslant t \leqslant \varepsilon^{\beta} t_{2}$

$$
f_{s}^{(N, n)}(t, s)<\delta .
$$

Let

$$
f_{a}^{(N)}(t, s)=\sum_{n=0}^{N} f_{i}^{(N, n)}(t, s)
$$

Then it follows from the last statement that if $\beta>N /(N+1)$, then for each $\delta>0$ and $t_{3} \in(s, T]$ there exists an $\varepsilon_{3}>0$ such that for $0<\varepsilon$ $\leqslant \varepsilon_{3}$ and $s \leqslant t \leqslant \varepsilon^{\beta} t_{3}$

$$
f_{\varepsilon}^{(N)}(t, 8)<\delta .
$$

On the other hand, the families $\left\{\varepsilon^{-N} U_{s}(t, s) g ; 0 \leqslant s \leqslant t \leqslant T\right\}$ and $\left\{\varepsilon^{-N} U_{e}(t, s) g ; 0 \leqslant s \leqslant t \leqslant T\right\}$ both tend to zero $\beta$-nearly uniformly on $(8, T]$. This means that for each $\delta>0$ and $\beta \epsilon(0,1)$ there exist a $t_{0}>8$ and an $\varepsilon_{0}>0$ such that for $0<\varepsilon \leqslant \varepsilon_{0}$ and $\varepsilon^{\beta} t_{0} \leqslant t \leqslant T$

$$
f_{\varepsilon}^{(N)}(t, 8)<\delta \text {. }
$$


The last two inequalities imply that the function $t \rightarrow f_{8}^{(N)}(t, s)$ tends to zero uniformly on $[s, T]$ for any $s \in[0, T)$, provided $0<\varepsilon \leqslant \varepsilon_{1}$, where $\varepsilon_{1}=\min \left(\varepsilon_{0}, \varepsilon_{3}\right)$. With Definition 5 and the definition of $f_{0}^{(N)}(t, s)$ this ends the proof of the lemma. QED.

The results obtained above will be summarized in the theorem.

THEOREM 3. Let the family $\{A(t) ; 0 \leqslant t \leqslant T\}$ and the function $t \rightarrow q(t)$ satisfy Assumption 1. Then the solution $\left\{x_{s}(t) ; 0 \leqslant t \leqslant T\right\}$ of the evolution equation

$$
\varepsilon \frac{d}{d t}\left(x_{\star}(t)\right)=A(t) x_{0}(t)+q(t)
$$

with the initial condition

$$
x_{s}(0)=\eta \in D(A)
$$

tends uniformly on $[0, T]$ faster than $e^{N}$ to the sum of the outer asymptotio solution $\left\{\bar{x}^{(N)}(t) ; 0 \leqslant t \leqslant T\right\}$ and the inner asymptotic solution $\left\{U^{(N)}(t, 0) \eta\right.$; $0 \leqslant t \leqslant T\}$, defined by Lemma 6 and Lemma 9 , respectively.

Comparison with the standard approach. The usual approach to the formal derivation of the equations in the singular perturbation method leads to results different from those obtained in this paper (see, for instance, [5]). In particular, the asymptotic solution consists of three terms: outer, inner and intermediate solutions. The inner solution contains some additional terms in comparison with the inner solution defined in this paper and the intermediate solution, obtained by matching the inner and outer solutions, has to be subtracted from the whole expression. For this reason, the singular perturbation method is sometimes referred to as the matched asymptotic expansion method.

In this section the standard perturbation procedure will be applied formally to the evolution equation with a small parameter considered in this paper and the resulting equations compared with those obtained previously.

Consider the evolution equation

$$
\varepsilon \frac{d}{d t}\left(x_{8}(t)\right)=A(t) x_{8}(t)+q(t)
$$

with the initial condition $x_{a}(0)=\eta$. To obtain the outer asymptotic solution, expand the function $x_{d}(t)$ into a truncated power series with respect to $\varepsilon$

$$
x_{e}(t)=\sum_{n=0}^{N} \varepsilon^{n} x_{n}(t)
$$


Substituting this series into the above equation and equating to zero terms containing the same powers of $\varepsilon$, one gets the system of equations

$$
\begin{gathered}
A(t) x_{0}(t)+q(t)=0 ; \\
\frac{d}{d t}\left(x_{n-1}(t)\right)=A(t) x_{n}(t) ; \quad n=1,2, \ldots, N .
\end{gathered}
$$

This shows that if the outer asymptotic solution is defined as a truncated series $\sum_{n=0}^{N} \varepsilon^{n} x_{n}(t)$, it is identical with the previously defined outer asymptotic solution.

To obtain the inner asymptotic solution, introduce a new independent variable $\tau=t / \varepsilon$ and a new function

$$
z_{\bullet}(\tau)=x_{\varepsilon}(\varepsilon \tau)
$$

which satisfies the evolution equation

$$
\frac{d}{d \tau}\left(z_{s}(\tau)\right)=A(\varepsilon \tau) z_{s}(\tau)+q(\varepsilon \tau)
$$

with the initial condition

$$
z_{e}(0)=\eta
$$

Expand the function $z_{\varepsilon}(\tau)$ into a truncated power series with respect to. 8 and $A(\varepsilon \tau)$ and $q(\varepsilon \tau)$ into a truncated Taylor series around $\tau=0$ :

$$
\begin{gathered}
z_{\varepsilon}(\tau)=\sum_{n=0}^{N} \varepsilon^{n} z^{(n)}(\tau) ; \\
A(\varepsilon \tau)=\left.\sum_{n=0}^{N} \frac{(\varepsilon \tau)^{n}}{n !} \frac{d^{n}}{d t^{n}}(A(t))\right|_{t=0} ; \\
q(\varepsilon \tau)=\left.\sum_{n=0}^{N} \frac{(\varepsilon \tau)^{n}}{n !} \frac{d^{n}}{d t^{n}}(q(t))\right|_{l=0} .
\end{gathered}
$$

Substituting these series into the last evolution equation and equating to zero terms containing the same powers of $\varepsilon$, one gets the following system of equations

$$
\begin{aligned}
\frac{d}{d \tau}\left(z^{(n)}(\tau)\right)=A(0) z^{(n)}(\tau)+ & \left.\sum_{k=1}^{n} \frac{\tau^{k}}{k !} \frac{d^{k}}{d t^{k}}(A(t))\right|_{t=0} \cdot z^{(n-k)}(\tau)+ \\
& +\left.\frac{\tau^{n}}{n !} \frac{d^{n}}{d t^{n}}(q(t))\right|_{t=0} ; \quad n=0,1, \ldots, N .
\end{aligned}
$$

with the initial conditions

$$
z^{(0)}(0)=\eta ; \quad z^{(1)}(0)=\ldots=z^{(N)}(0)=0 .
$$


Returning to the variable $t$ and writing $z^{(n)}(t / \varepsilon)=\tilde{x}_{e}^{(n)}(t)$, one gets the system of equations.

$$
\begin{aligned}
\varepsilon \frac{d}{d t}\left(\tilde{x}_{s}^{(n)}(t)\right)=A(0) \tilde{x}_{e}^{(n)}(t) & +\left.\sum_{k=1}^{N}\left(\frac{t}{\varepsilon}\right)^{k} \frac{1}{k !} \frac{d^{k}}{d t^{k}}(A(t))\right|_{t=0} \cdot \tilde{x}_{s}^{(n-k)}(t)+ \\
& +\left.\left(\frac{t}{\varepsilon}\right)^{n} \cdot \frac{1}{n !} \frac{d^{n}}{d t^{n}}(q(t))\right|_{t=0} ; \quad n=0,1, \ldots, N ;
\end{aligned}
$$

with the initial conditions

$$
\tilde{x}_{e}^{(0)}(0)=\eta ; \quad \tilde{x}_{s}^{(1)}(0)=\ldots=\tilde{x}_{s}^{(N)}(0)=0 .
$$

Thus it is seen that if the inner asymptotic solution is defined as a truncated series $\sum_{n=0}^{N} \varepsilon^{n} \tilde{x}_{s}^{(n)}$, it differs from that introduced previously.

The difference is called the intermediate asymptotic solution and it should be equal to the expansion of the outer asymptotic solution for small times. Such property for the zero and first order expansions was proved in [3]-[5]. In this sense the inner and outer asymptotic solutions match each other. The asymptotic solution tending to the exact solution uniformly in the interval is then obtained as the sum of the inner and outer asymptotic solutions minus the intermediate solution.

Thus it is seen that the singular perturbation procedure developed rigorously in this paper leads in practical applications to equations simpler than those obtained formally by the application of the standard perturbation approach. In particular, the matching procedure for evolution equations is in fact not necessary and can be avoided by making use of the present approach.

\section{References}

[1] J. D. Cole, Perturbations methods in applied mathematics, Blaisdell, Waltham 1968.

[2] N. Dunford and J. T. Schwartz, Linear operators, Part I, Interscience.Publishers, New York 1958.

[3] W. L. Hendry, Application of the method of matched asymptotic expansions to a problem in linear transport theory, J. Math. Phys. 11 (1970), p. 1743-1749.

[4] - Solution to the linear time-dependent neutron transport equation with time-dependent source and cross sections, Nuclear Science and Engineering 45 (1971), p. 1-6.

[5] - and G. I. Bell, An analysis of the time-dependent neutron transport equation with delayed neutrons by the method of matched asymptotic expansions, ibidem 35 (1960), p. 240-248.

[6] T. Kato, Integration of the equation of evolution in a Banach space, J. Math. Soc. Japan 5 .(1953), p. 208-234. 
[7] J. Kisy ński, Sur les opérateur de Green des problèmes de Cauchy abstraits, Studia Math. 23 (1964), p. 285-328.

[8] S. G. Krein, Linear differential equations in a Banach space, Nauka, Moscow 1967 (in Russian).

[9] R. O. O'Malley, Topics in singular perturbations, Advances Math. 2 (1968), p. $365-470$.

[10] J. Mika, Singular perturbation method in neutron transport theory, J. Math. Phys. 15 (1974), p. 892-898.

[11] - Singular perturbation method for linear differential equations in Banach space, Math. Balcanica 3 (1973), p. 321-334.

Regu par la Rédaction le 28.1.1974 